\title{
The effect of a seven-week exercise program on golf swing performance and musculoskeletal measures
}

2016, Vol. II(4) 610-618

(C) The Author(s) 2016

Reprints and permissions:

sagepub.co.uk/journalsPermissions.nav

DOI: $10.1177 / 1747954$ | 16654784

spo.sagepub.com

\author{
Mico H Olivier', Sean A Horan², Kerrie A Evans ${ }^{2}$ \\ and Justin WL Keogh ${ }^{1,3,4}$
}

\begin{abstract}
As most golf exercise studies have shown improved golf performance as a result of two or three sessions per week, the present study investigated the effects of a supervised exercise session performed once a week for seven weeks on golf swing variables and musculoskeletal screening measures. Professional Golfers Association of Australia International Golf Institute student golfers $(n=43)$ with a mean \pm standard deviation handicap of $8.6 \pm 8.3$ participated in the study. Each golfer performed 10 musculoskeletal tests and a standardised 60-shot golf performance test (TrackMan, Vedbaek, Denmark) on separate days before and after the seven-week program. Significant improvements in a number of musculoskeletal tests (i.e. left leg bridging $(6.6 \%)$, thoracic extension $(62.5 \%)$, right thoracic rotation (23.3\%), and right $(20.8 \%)$ and left single leg squat $(29.1 \%)$ ) were observed (all $p \leq 0.024)$; however, no significant differences were observed for any golf swing variables. Future research investigating different training protocols may help to determine whether the type or frequency of training has the greatest influence on golf swing performance.
\end{abstract}

\section{Keywords}

Golf swing, fitness, musculoskeletal screening, training, Trackman

\section{Introduction}

Golf has traditionally been viewed as a skill-based sport that is not overtly physical and has a low risk for injury. However, this view is in contrast to elite golf practice with recent reviews finding high levels of lower body, trunk and upper body muscle activity during the golf swing, ${ }^{1}$ relatively high physiological stress during match play ${ }^{2}$ and relatively high injury rates to areas such as the lower back, shoulder and wrist. ${ }^{3-5}$ Most golf-related injuries are thought to result from the large number of practice hours golfers perform, ${ }^{3-6}$ the asymmetrical nature of the golf swing ${ }^{7}$ or biomechanical-related swing inefficiencies. ${ }^{3}$

As a result, more golfers are performing strength and conditioning exercise programs in an attempt to reduce the risk of injury and improve their physical fitness capacities, which may in turn aid golf competitive performance. Several studies have examined the effects of exercise programs on physical characteristics and found pronounced improvements in muscular strength, power, endurance and range of motion in golfers of various ages and skill levels. ${ }^{8-13,15}$ For example, Doan et al. ${ }^{8}$ found that highly skilled golfers significantly $(p<0.05)$ improved their relative strength and flexibility by $7 \%-24 \%$ and $7 \%-16 \%$, respectively, after 11 weeks of resistance training. The majority of previous exercise studies have ranged between 8 and 18 weeks and typically involved two to three exercise sessions per week. None of these golf exercise studies have currently involved less than two training sessions per week involving golfers of various ages. Further, only

Reviewers: Harvey Newton (Newton Sports, USA) Sam Robertson (Victoria University, Australia)

'Faculty of Health Science and Medicine, Bond University, Gold Coast, QLD, Australia

${ }^{2}$ School of Allied Health Sciences, Menzies Health Institute Queensland, Griffith University, Gold Coast, QLD, Australia

${ }^{3}$ Sport Performance Research in New Zealand (SPRINZ), AUT University, Auckland, New Zealand

${ }^{4}$ Cluster for Health Improvement, Faculty of Science, Health, Education and Engineering, University of the Sunshine Coast, Sippy Downs, QLD, Australia

\section{Corresponding author:}

Mico H Olivier, Faculty of Health Sciences and Medicine, Bond University, University Drive, Gold Coast, QLD 4026, Australia.

Email: mico.olivier@student.bond.edu.au 
Larkin et al., ${ }^{13}$ Fradkin et al., ${ }^{14}$ Reyes ${ }^{15}$ and Lamberth et al. ${ }^{16}$ have involved training programs of less than eight weeks, utilising programs that were only three, five, six and seven weeks in duration, respectively.

Despite growing evidence that exercise offers golfers important performance benefits, ${ }^{8-12}$ the minimum duration and frequency of golf exercise programs to achieve significant improvements in physical fitness and golf performance is still unknown. This is an important question as golf can be a time-intensive sport, and many golfers have considerable work, study and/or family commitments. These demands may mean that it is difficult for many golfers to apportion enough time to be able to perform two to three exercise sessions per week as has been performed in previous intervention studies. ${ }^{8-12,17}$ Given little research has been conducted that has examined the effects of a reduced exercise training duration and/or frequency on golfers, such research appears warranted. Previous studies that have examined reduced training frequency ${ }^{18-20}$ have demonstrated that relatively untrained older adults can substantially improve muscular strength, power and/or endurance with one exercise session per week. Furthermore, meaningful body composition and physical performance gains can be observed in as little as four weeks of training in professional rugby union players. ${ }^{21}$ It would therefore appear possible that even one resistance exercise session per week may induce pronounced changes in muscular strength, power and endurance that may subsequently improve musculoskeletal screening test outcomes and/or golf performance in golfers with little to no physical conditioning experience.

Strength and conditioning coaches and physiotherapists frequently employ musculoskeletal screening assessments ${ }^{22-27}$ to assess physical fitness deficiencies and movement dysfunctions thought to contribute to reduced performance or an increase injury risk in athletes. Parsonage et al. ${ }^{26}$ investigated elite adolescent rugby players and divided them into three groups based on four Conditioning Specific Movement Tasks. The two groups who performed better on the movement competency tests, thought to be specific for rugby, were significantly faster over 10,20 and $40 \mathrm{~m}$, jumped significantly higher, and covered significantly greater distances on the Yo-Yo intermittent recovery level 1 than the group who scored lower on these movement competency tests.

However, some limitations still exist with the common screening assessments. First, there is currently limited evidence as to the efficacy of these screening protocols in reducing the risk of injury. Secondly, these screening approaches appear quite generic and have focused on athletes competing in running-based sports. On this basis, a more 'golf-specific' musculoskeletal test protocol referred to as the Ten Test on Range
(The Golf Athlete, Brisbane, Australia) assessment protocol has been developed. This screening tool involves a series of tests designed to assess physical parameters thought to be important for golf and thus was adopted in this study. ${ }^{28}$ This protocol is commonly used by many Australian golf professionals and physiotherapists, with many of the assessments in this battery reported to have good intra- and inter-rater reliability when used by inexperienced and experienced testers. ${ }^{29}$

Therefore, the aim of the current study was to investigate the effects of a short duration (i.e., seven-week) exercise program comprising one exercise session per week on musculoskeletal test scores and golf swing performance. It was hypothesised that the exercise program would result in improvements in both musculoskeletal screening scores and golf swing performance.

\section{Methods}

\section{Participants}

Forty-three golf students (7 females and 36 males, mean \pm SD age $24 \pm 8.9$ years) were recruited to participate in the study (handicap, $8.6 \pm 8.3$ strokes). All students were enrolled in a Diploma of Golf Management at the PGA-IGI (Professional Golf AssociationInternational Golf Institute), which is a full-time, one year golf management course. All participants had a minimum of one years' golf experience, and reported little, if any, previous experience with an exercise program. Written consent was obtained prior to data collection, and approval to conduct the study was given by the Bond University Human Research Ethics Committee and Griffith University Human Research Ethics Committee.

\section{Overview of experimental design}

The current project was a single-group-based intervention study involving a pre-post design. Prior to the seven-week exercise intervention, participants completed assessments of musculoskeletal screening and golf performance across two testing sessions. At the first session, participants were asked to perform a series of 10 selected musculoskeletal screening tests, ${ }^{28}$ while at the second session participants performed a standardised, field-based golf performance test which included 60 golf shots (Combine Test, TrackMan, ISG A/S, Denmark). Following baseline testing, all participants undertook a once-weekly, seven-week exercise program consisting of muscular strength and endurance exercises. Following completion of the exercise program, all participants repeated the same musculoskeletal screening and golf swing performance tests performed at baseline. 


\section{Musculoskeletal screening assessments}

The present study employed 10 musculoskeletal screening tests to examine flexibility, muscular endurance and movement competency of all participants. The testing sessions were conducted at a golf club, with each of the musculoskeletal screening sessions lasting $\sim 1 \mathrm{~h}$, whilst each combine performance test taking $\sim 45 \mathrm{~min}$. An experienced exercise scientist, two experienced sports physiotherapists, 10 post-graduate physiotherapy students and a Master of Science student conducted the musculoskeletal testing sessions at the country club. The combine test was performed on the golf range under the supervision of a Master of Science student with the assistance of a golf lecturer at the country club. Table 1 provides a description and rationale of the musculoskeletal screening tests utilised in this study. As already stated and based on data from a previous study, ${ }^{29}$ many of the musculoskeletal screening tests used in the current study have been shown to have good inter- and intra-rater reliability. Specifically, the following tests have been found to have good interrater reliability: seated trunk rotation $(\mathrm{ICC}=0.60$ ); lift off (thoracic extension) ( $\mathrm{ICC}=0.67)$ and overhead squat ( $\mathrm{ICC}=0.85$ ); while the following tests have been found to have fair inter-rater reliability: single leg squat $(\mathrm{ICC}=0.45)$ and single leg bridge $(\mathrm{ICC}=0.35)$. Further, the following tests have been found to have excellent-good intra-tester reliability: seated trunk rotation ( $\mathrm{ICC}=0.58-0.97$ ); lift off (thoracic extension) ( $\mathrm{ICC}=0.63-0.98)$; overhead squat $(\mathrm{ICC}=0.69-0.99)$; while the following tests have been found to have excellent-fair intra-rater reliability: single leg squat $(\mathrm{ICC}=0.28-0.92)$ and single leg bridge $(\mathrm{ICC}=$ $0.34-0.95){ }^{29}$

\section{Golf swing performance}

Golf swing performance was measured using a TrackMan (TrackMan IIIe, Vedbaek, Denmark) Doppler Radar system. While the validity of the TrackMan system remains to be determined, it has previously been used by researchers ${ }^{31}$ and is commonly used by Australian golf coaches and on the USA and European professional golf tours. ${ }^{32}$ Using this device enables participants to complete a field-based golf performance test called the 'combine test' (TrackMan Performance Studio Version 3.0, TrackMan, Denmark), which involved 60 shots to 10 standardised targets. For each shot, the software provides a score from 0 to 100 based on distance from the target along the target line and lateral deviation from the target line. The lowest score for a shot equates to 0 and the highest 100. Distance markers were placed at 50, 100 and $150 \mathrm{~m}$, with the markers measured using a golf laser range finder (Bushnell Medalist, Overland Park, USA).
The distance markers were placed in a line, providing each golfer with a target line to hit towards. All testing was performed on an outdoor driving range $(150 \times 300 \mathrm{~m})$, with each participant hitting shots from an artificial grass mat. Environmental conditions were similar on all testing days, with temperatures between 24 and $28^{\circ} \mathrm{C}$ and light winds between 10 and $20 \mathrm{~km} \mathrm{~h}^{-1}$. The golf balls used were range balls (Srixon, Sydney, Australia), which generally travel $\sim 80 \%$ of the distance of a standard competition ball. The TrackMan device was set up 1.5-2.0 m behind the ball and in line with the target line similar to the recommendations of Robertson et al. ${ }^{31}$

Following a warm-up and familiarisation period of 15-20 min which included shots with a selection of clubs (i.e., self-selected), participants undertook the combine test. The test requires the golfer to hit shots using their club of choice (except the maximum distance shot where a driver is used) to distances along a target line at $65,75,85,95,105,125,145$ and $165 \mathrm{~m}$, as well as a shot for maximum distance with the driver club. At each distance the golfer hits three shots starting at the $65 \mathrm{~m}$ distance, followed by three shots to each subsequent distance thereafter. At the completion of these 30 shots, the process is repeated a second time until a total of 60 shots is completed. In the current study, participants were instructed to hit the ball as straight and close as possible to the target distance, except for the driver where participants were instructed to hit the ball as far and straight as possible. In addition to a score for each shot and an overall score out of 100, the TrackMan device allows for the measurement of a range of other ball striking performance variables. In the current study, the measurements we were interested in were club head speed (CHS), ball speed, carry distance, total distance, carry side (lateral deviation distance) and smash factor (ball speed/clubhead speed). A description of the variables is given in Table 2. Due to the distance and accuracy requirements of the driver shots, we also felt that the outcomes we selected from the TrackMan data of the driver shots were more reflective of the real requirements of driving during golf competition, whereby hitting the ball a large distance accurately is an important component of golf performance. We therefore thought it was more likely that the strength and conditioning program would influence these selected variables more so than the other outputted TrackMan variables.

\section{Exercise program}

All participants were asked to complete one supervised exercise class per week for a seven-week period. The exercise program (see Table 3) was developed through consensus moderation between all researchers 
Table I. A description of the musculoskeletal screening tests.

$\begin{array}{ll}\begin{array}{l}\text { Musculoskeletal } \\ \text { screening test }\end{array} & \begin{array}{l}\text { Structures assessment } \\ \text { Side bridge (s) }\end{array} \\ \begin{array}{l}\text { Challenges the muscles of the } \\ \text { anterolateral chest } \\ \text { wall (lateral trunk flexors), the } \\ \text { quadratus lumborum, } \\ \text { external and internal obliques. }\end{array} \\ \begin{array}{l}\text { Front plank (s) } \\ \text { Challenges the muscles of the } \\ \text { anterior abdominal muscles. }\end{array} \\ \begin{array}{l}\text { Combined elevation } \\ \text { (thoracic extension) }(\mathrm{cm})\end{array} \\ \begin{array}{l}\text { Global measure of the flexibility } \\ \text { of the upper back and thor- } \\ \text { acic spine }\end{array}\end{array}$

Hip internal rotation $\left({ }^{\circ}\right)$

Hip external rotation $\left({ }^{\circ}\right)$

Straight leg raise $\left(^{\circ}\right)$

Thoracic rotation $\left({ }^{\circ}\right)$
Global measure of hip internal rotation movement

Global measure of hip external rotation movement

Global measure of the flexibility of the hamstring muscle group

Global measure of the flexibility of the cervical \& thoracic spine
Protocol

Participants laid on their side on the floor, with their forearm and feet supporting their weight. The elbow joint of the support arm flexed at $90^{\circ}$, with the opposite arm placed across their chest and legs extended. Participants then elevated their hips and kept a straight line with their whole body for maximum time, as assessed by a stopwatch., 7,29

Participants were prone, placing their hands and elbows in front of them on the ground. Participants had to elevate their bodies to start the test, using their hands and toes as pivots whilst maintaining a straight, neutral body position where time taken was in seconds, and participants were encouraged to maintain this for as long as possible.

Participants were prone on the floor; arms extended out in front of them with hands pointing forward, palms facing down and thumbs touching. Participants kept their chins on the floor, looking up at their thumbs with their chest, hips and feet kept on the floor during the test. They had to then lift up their arms slowly and as far as possible whilst keeping thumbs touching. The vertical distance from their hands to the floor was taken with a tape measure.

Participants were supine on a plinth; a belt was around their hips at the anterior superior iliac spine to stabilise their pelvis. The non-test leg was kept straight, and the stretching leg was in $90^{\circ}$ hip flexion. The assessor inwardly rotated the knee which outwardly rotated the foot until resistance. The stationary arm of the goniometer was in line with trunk and the movable arm in line with tibia. The Centre of rotation (COR) was at the central patella tendon/inferior pole (central knee joint). This was repeated on the other leg.

Participants were supine on a plinth; a belt was placed around their hips at the anterior superior iliac spine to stabilise their pelvis. With one leg straight, the other leg was at $90^{\circ}$ hip flexion. Their foot was outwardly rotated which inwardly rotated the hip until resistance. The stationary arm of the goniometer was in line with trunk and the movable arm in line with tibia. The COR was at central patella tendon/inferior pole (central knee joint). This was then repeated on the other leg.

Participants were supine on a plinth with hands by their sides. One instructor lifted the test leg extended into hip flexion, keeping the knee extended until resistance was felt or pain initiated. The opposite leg and pelvis was kept straight by another instructor. An inclinometer was placed on the anterior part of the tibia, measuring the degrees of flexibility.

Participants sat upright in a chair. A golf club was placed behind their backs for them to cradle the club in their elbows and maintaining club-spine contact. Participants kept knees, feet and hips facing forward. Participants rotated to one side as far as possible until club-spine contact was no longer maintained. A goniometer measured degrees of rotation by placing the stationary arm above the participants head with the moving arm in line with the leading shoulders direction and shaft direction. This was repeated on the other side. 
Table I. Continued

\begin{tabular}{ll}
$\begin{array}{l}\text { Musculoskeletal } \\
\text { screening test }\end{array}$ & Structures assessment \\
\hline Overhead squat $(I-5)$ & $\begin{array}{c}\text { Global measure of upper back, } \\
\text { hip and ankle mobility }\end{array}$
\end{tabular}

Single leg bridging lift (I-5) Global measure of hip strength and weight transference

Single leg squat (I-5)
Global measure of hip \& trunk muscle strength
Protocol

Participants stood feet shoulder width apart and were instructed to squat down until thighs were parallel to the floor. They had to keep the golf club above their head. A score of I indicated poor performance, whereby the participant could get their thighs to parallel with their arms across their chest, all the way up to 5 , where the participant could squat with thighs parallel and arms extended above head.

Participants were instructed to lie supine on the floor, place feet apart, and lift their hips off the ground whilst extending one leg and holding it for $10 \mathrm{~s}$. Participants were scored on a I-5 scale, with I being the inability to straighten the leg and 5 is a steady $10 \mathrm{~s}$ hold with no cramping in the hamstring muscles.

Participants folded their arms across their chest, stood on one leg with the other leg out in front. Participants were encouraged to do six controlled squats whilst aiming to reach $90^{\circ}$ knee flexion. A scoring system of I, significant dropping of hips, knees and losing balance, to 5 , performed squat with good control to $90^{\circ}$ knee flexion, was used.

COR: Centre of rotation.

Table 2. Definitions of multiple golf performance variables as measured by the TrackMan Pro.

\begin{tabular}{ll}
\hline Golf performance variables & Definitions \\
\hline Club head speed (CHS) (TrackMan website) & The speed at which the club head is moving immediately before impact. \\
Ball speed (TrackMan website) & The speed of the golf ball immediately after impact is made. \\
Carry distance ('flat') (TrackMan website) & $\begin{array}{l}\text { The distance the ball travels through the air. } \\
\text { Carry side (accuracy) }\end{array}$ \\
& $\begin{array}{l}\text { Measured in relation to the target line with a positive reading referring to } \\
\text { metres landed to the right of the target and a negative reading } \\
\text { meaning metres landed to the left. }\end{array}$ \\
Smash factor (TrackMan website) & The ball speed divided by club speed. \\
\hline
\end{tabular}

(all of which were trained exercise scientists or physiotherapists), with consideration given to the biomechanics and physical requirements of the golf swing, common injury sites of golfers, as well as the practicalities of the exercise facility and time available per class. The classes were held in a group exercise room and supervised by an experienced sports physiotherapist and a Master of Science student. The available equipment consisted of bikes, step-up boxes, a smaller barbell and weight plates up to $5 \mathrm{~kg}$. Each class ran for approximately $60 \mathrm{~min}$ which included a 5-min warm-up and cool-down component. Consequently, the exercise program consisted of several traditional muscular endurance exercises as well as more golfrelated movements involving rotational movements of the hips, torso and shoulders. ${ }^{1}$ Exercises were adapted and progressed or alternatively regressed to suit the physical levels of each participant as determined using the baseline musculoskeletal screening assessment.

\section{Statistical analysis}

As assumptions of normality were met for all outcomes based on the Shapiro-Wilks test, means and standard deviations were used as measures of centrality and spread. A repeated measures analysis of variance was performed to evaluate pre-post differences in all outcomes for the entire group. However, because of considerable variation in the number of exercise sessions attended by some participants, a secondary analysis was performed comparing the training response of those completing six or greater sessions $(n=11)$, those 
Table 3. A list of exercises implemented in the seven-week exercise program.

\begin{tabular}{|c|c|c|c|c|}
\hline Body part & $\begin{array}{l}\text { Exercises } \\
\text { progressions }\end{array}$ & Sets & Reps & $\begin{array}{l}\text { Rest } \\
\text { between } \\
\text { sets }\end{array}$ \\
\hline Lower body & $\begin{array}{l}\text { Bodyweight lunge } \\
\text { Bodyweight lunges } \\
\text { with twist } \\
\text { Weighted lunges } \\
\text { with twist } \\
\text { Hockey jumps }\end{array}$ & 3 & $8-12$ & $45-60 s$ \\
\hline Upper push & $\begin{array}{l}\text { Bodyweight push } \\
\text { ups on knees } \\
\text { Bodyweight push ups } \\
\text { Dumbbell push ups }\end{array}$ & 3 & $8-12$ & $45-60 s$ \\
\hline Core & $\begin{array}{l}\text { Prone hold } \\
\text { Seated Russian twist } \\
\text { Barbell woodchop }\end{array}$ & 3 & $\begin{array}{l}45 \mathrm{~s} \\
1 \mathrm{~min} \\
10-15 \\
10-15\end{array}$ & $45-60 s$ \\
\hline Upper pull & $\begin{array}{l}\text { Prone one-arm } \\
\text { dumbbell rows } \\
\text { Renegade row }\end{array}$ & 3 & $8-12$ & $45-60 s$ \\
\hline Sprints & $\begin{array}{l}\text { Resisted band } \\
\text { sprints }\end{array}$ & I & 5 & $\begin{array}{r}\text { Walk back } \\
\text { to start }\end{array}$ \\
\hline
\end{tabular}

who completed four sessions $(n=8)$ and those who completed two or less sessions $(n=10)$. The three groups were referred to as the High, Moderate and Low adherence groups, respectively. Given many of the outcomes for this sub-group, analysis did not meet the assumptions of normality according to the Shapiro-Wilks tests, a Mann-Whitney U test was performed to compare the pre-post change scores between the sub-groups for all outcome measures. All statistical analyses were performed in SPSS Version 20, with significance set at $p<0.05$.

\section{Results}

Thirty-four of the original 43 participants completed baseline and post-testing, and the data from these 34 participants are presented in Tables 4 and 5. Adherence data were also recorded for every exercise session, with a mean attendance rate of $55 \%$ (i.e., an average of 3.8 out of 7 sessions was attended by the whole group). As shown in Table 4, significant improvements in a number of musculoskeletal screening tests were observed for the entire group including: left leg bridging $(6.6 \%, p=0.024)$; combined elevation (thoracic extension) $62.5 \%, p=0.013)$; right thoracic rotation $(23.3 \%$, $p<0.001)$ and right and left single leg squat performance $(20.8 \%, p=0.012$ and $29.1 \%, p=0.001$, respectively). In contrast, no significant $(p>0.05)$ changes in
Table 4. Changes in musculoskeletal screening test scores after the seven-week training program.

\begin{tabular}{|c|c|c|c|}
\hline Outcome & $\begin{array}{l}\text { Baseline } \\
(\text { mean } \pm S D)\end{array}$ & $\begin{array}{l}\text { Post } \\
(\text { mean } \pm S D)\end{array}$ & $p$ value \\
\hline \multicolumn{4}{|l|}{ Side bridge (s) } \\
\hline Right & $75 \pm 34.8$ & $87 \pm 34.7$ & 0.146 \\
\hline Left & $75 \pm 30.9$ & $93 \pm 39.6$ & 0.065 \\
\hline \multicolumn{4}{|l|}{ Bridging leg lift $(\mathrm{I}-5)$} \\
\hline Right & $4.6 \pm 0.7$ & $4.8 \pm 0.5$ & 0.570 \\
\hline Left & $4.5 \pm 0.7$ & $4.8 \pm 0.4$ & $0.024 *$ \\
\hline \multicolumn{4}{|l|}{ Straight leg raise $\left({ }^{\circ}\right)$} \\
\hline Right & $68 \pm 11.5$ & $69 \pm 12.7$ & 0.691 \\
\hline Left & $69 \pm 11.3$ & $70 \pm 11.9$ & 0.679 \\
\hline \multicolumn{4}{|l|}{ Hip internal rotation $\left({ }^{\circ}\right)$} \\
\hline Right & $27 \pm 10.2$ & $26 \pm 6.8$ & 0.625 \\
\hline Left & $28 \pm 9.1$ & $25 \pm 7.8$ & 0.224 \\
\hline \multicolumn{4}{|l|}{ Hip external rotation $\left({ }^{\circ}\right)$} \\
\hline Right & $40 \pm 10.2$ & $42 \pm 12.6$ & 0.553 \\
\hline Left & $39 \pm 10.8$ & $39 \pm 14.4$ & 0.796 \\
\hline $\begin{array}{l}\text { Combined elevation } \\
\text { (thoracic } \\
\text { extension) }(\mathrm{cm})\end{array}$ & $8 \pm 6.7$ & $13 \pm 8.9$ & $0.013 *$ \\
\hline \multicolumn{4}{|l|}{ Thoracic rotation $\left({ }^{\circ}\right)$} \\
\hline Right & $30 \pm 6.2$ & $37 \pm 7.4$ & $<0.001 * *$ \\
\hline Left & $3 \mathrm{I} \pm 7.5$ & $30 \pm 9.6$ & 0.860 \\
\hline Overhead squat (I-5) & $3.8 \pm 1.6$ & $4.2 \pm 1.2$ & 0.354 \\
\hline \multicolumn{4}{|l|}{ Single leg squat $(I-5)$} \\
\hline Right & $2.4 \pm 0.7$ & $2.9 \pm 0.9$ & $0.012^{*}$ \\
\hline Left & $2.4 \pm 0.8$ & $3.1 \pm 0.8$ & $0.001 *$ \\
\hline Front plank (s) & $140 \pm 69.7$ & $152 \pm 79.6$ & 0.507 \\
\hline
\end{tabular}

*Statistical significance level at $p<0.05$; * Statistical significance level at $p<0.00$ I.

Table 5. Changes in golf swing performance after the sevenweek training program.

\begin{tabular}{lrcc}
\hline Outcome & \multicolumn{1}{l}{$\begin{array}{l}\text { Baseline } \\
(\text { mean } \pm S D)\end{array}$} & $\begin{array}{l}\text { Post } \\
(\text { mean } \pm \text { SD) }\end{array}$ & $p$ value \\
\hline Combine test & $66.0 \pm 12.0$ & $67.1 \pm 9.4$ & $0.68 I$ \\
$\begin{array}{l}\text { Club head speed } \\
\quad\left(\mathrm{km} \mathrm{h}^{-1}\right)\end{array}$ & $162.1 \pm 17.5$ & $162.1 \pm 17.0$ & 0.995 \\
Ball speed $\left(\mathrm{km} \mathrm{h}^{-1}\right)$ & $225.7 \pm 25.7$ & $224.1 \pm 24.0$ & 0.773 \\
Smash factor & $1.39 \pm 0.05$ & $1.38 \pm 0.05$ & 0.391 \\
Driving distance $(\mathrm{m})$ & $234.6 \pm 37.3$ & $220.3 \pm 31.1$ & 0.072 \\
Carry side $(\mathrm{m})$ & $0.6 \pm 12.4$ & $0.5 \pm 13.2$ & 0.718 \\
\hline
\end{tabular}

any of the golf performance measures were observed for the entire group (see Table 5).

Results of the Mann-Whitney U test for the effect of attendance on outcome measures revealed a small 
number of significant between-group effects. These included an improved left-side bridge holding time for the High versus the Low group (25.2 seconds, $p=0.003$ ); improved overhead squat performance for the High versus Moderate group ( 0.6 units out of 5, $p=0.035)$ and an improved left side bridge holding time for the Moderate versus the Low group (13.3s, $p=0.043$ ). For golf swing performance measures (i.e., combine test overall scores, clubhead speed, ball speed, driving distance, smash factor and carry side), no significant between-group differences in change scores were observed between the three attendance groups.

\section{Discussion}

The main findings of this study were that substantial improvements were observed in several musculoskeletal screening tests including the left leg bridging lift, combined elevation, right thoracic rotation and right and left single leg squat performance. However, no significant improvements for any of the golf swing performance variables were observed. Additionally, Mann-Whitney $\mathrm{U}$ test results demonstrated no meaningful differences in the change scores between the three attendance groups for any of the golf swing performance measures. However, more frequent training was associated with greater improvements in a number of the musculoskeletal screening tests including the left-side bridge and overhead squat.

Improvements in a number of the musculoskeletal screening tests as a result of the exercise intervention provide partial support for our first hypothesis. Such findings are consistent with several previous investigations, all of which found pronounced improvements in strength and range of motion outcomes when golfers engaged in a regular exercise program. ${ }^{8,10-12}$ For example, Doan et al. ${ }^{8}$ assessed one-repetition maximum (1RM) strength for the bench press, shoulder press, lat pulldown and squat exercises which are well-known global exercises typical of strength training, and reported significant improvements in all these tests following an 11-week exercise intervention. Their clubhead speed changes were not significant either, reporting increases of $3.4 \%$ for women and $0.6 \%$ for men. A different approach was used by Lephart et al. ${ }^{12}$ assessing strength of the torso, shoulders and hip muscles using a Biodex System III Multi-Joint testing and Rehabilitation System. Following the eight-week exercise intervention, Lephart et al. ${ }^{12}$ reported significant improvements in torso rotational and hip abduction strength as well as all range of motion variables. Their result also included ball velocity, carry distance and total distance improvements by $5.0 \%, 7.7 \%$ and $6.8 \%$, respectively. Both the aforementioned studies found superior golf performance changes in comparison to our study which illustrated the following: no change in clubhead speed, a $0.7 \%$ decrease in ball speed and a $6 \%$ decrease in driving distance. These unfavourable outcomes may be attributed to the nature of the training sessions being only performed once a week due to their wide range of study, work, golf and personal commitments.

A unique feature of the current study was the frequency of sessions performed each week (i.e., one session). Previous studies that have examined the effect of a single exercise session per week on previously untrained older adults found significant improvements in a range of physical qualities including muscular endurance and functional performance. ${ }^{18-20}$ Similarly, we found improvements in several range of motion and movement competency tests in untrained golfers. Despite improvements in physical competency, this current study along with Lamberth et al. ${ }^{16}$ observed no translation into improved golf performance, which is in contrast to the observed increases in both physical qualities (leg strength, core strength, range of motion) and CHS reported in previous studies. ${ }^{8-12,17}$ The most plausible explanation for the discrepancy is that most previous golf exercise programs have utilised two to three sessions per week over 8-18 weeks, whereas our study consisted of only one session per week for seven weeks. Limited improvement in golf performance may reflect a minimum dosage effect, whereby a greater training duration might be required if the frequency is only once per week. This view is partially supported by the results of the sub-analysis comparing the High, Moderate and Low attendance groups. The effect of attendance on the measured outcomes provided valuable information, supporting the assumption that with greater compliance during the exercise program, greater changes in physical movement characteristics can be expected.

There were several limitations that warrant acknowledgement in this study. Firstly, no control group was used during the study. Second, exercise program adherence rates were lower than previous studies, with a mean adherence rate of $55 \%$ for the whole group over the seven-week period. This is likely to have decreased the overall effectiveness of the intervention. While individualised training programs were considered, they were deemed not to be feasible due to limitations in access to the training facility and the prohibitive cost of providing individual supervised sessions. Consequently, we utilised groups sessions that contained individually focussed exercises, which may have diluted the overall effectiveness of the program. Lastly, some of the musculoskeletal screening tests used in this study have been shown to have only fair or moderate reliability (e.g., single leg bridge and single leg squat). Some caution must therefore be utilised 
when interpreting the significance of the trainingrelated changes in these outcomes within the current study.

\section{Conclusion}

Our results indicate that although exercising once a week for seven weeks leads to no meaningful improvement in golf swing performance as assessed by the combine test, it did result in substantial improvements in several musculoskeletal screening tests. In relation to the wider golf strength and conditioning literature, our results suggest that in relatively untrained golf populations some improvements in physical characteristics may occur with short duration, once a week training, but a longer duration or frequency of exercise is likely needed to improve golf swing performance. Future research should directly compare variations in exercise duration or frequency using randomised controlled trial designs to better elucidate the effect of manipulating exercise prescription variables on musculoskeletal screening and golf performance outcomes.

\section{Declaration of Conflicting Interests}

The author(s) declared no potential conflicts of interest with respect to the research, authorship, and/or publication of this article.

\section{Funding}

The author(s) received no financial support for the research, authorship, and/or publication of this article.

\section{References}

1. McHardy A and Pollard H. Muscle activity during the golf swing. Br J Sports Med 2005; 39: 799-804.

2. Farrally MR, Cochran AJ, Crews DJ, et al. Golf science research at the beginning of the twenty-first century. J Sports Sci 2003; 21: 753.

3. Batt ME. A survey of golf injuries in amateur golfers. Br J Sports Med 1992; 26: 63-65.

4. Gosheger G, Liem D, Ludwig K, et al. Injuries and overuse syndromes in golf. Am J Sports Med 2003; 31: 438-443.

5. McHardy A, Pollard H and Luo K. Golf injuries: a review of the literature. Sports Med 2006; 36: 171-187.

6. Lindsay DM, Versteegh TH and Vandervoort AA. Injury prevention: avoiding one of golf's more painful hazards. Int J Sports Sci Coach 2009; 4: 129-148.

7. Evans K, Refshauge KM, Adams R, et al. Predictors of low back pain in young elite golfers: a preliminary study. Phys Ther Sport 2005; 6: 122-130.

8. Doan BK, Newton RU, Kwon Y-H, et al. Effects of physical conditioning on intercollegiate golfer performance. $J$ Strength Cond Res 2006; 20: 62-72.

9. Fletcher IM and Hartwell M. Effect of an 8-week combined weights and plyometrics training program on golf drive performance. J Strength Cond Res 2004; 18: 59-62.
10. Thompson CJ and Osness WH. Effects of an 8-week multimodal exercise program on strength, flexibility, and golf performance in 55- to 79-year-old men. J Aging Phys Activ 2004; 12: 144-156.

11. Thompson CJ, Cobb KM and Blackwell J. Functional training improves club head speed and functional fitness in older golfers. J Strength Cond Res 2007; 21: 131-137.

12. Lephart SM, Smoliga JM, Myers JB, et al. An eight-week golf-specific exercise program improves physical characteristics, swing mechanics, and golf performance in recreational golfers. J Strength Cond Res 2007; 21: 860-869.

13. Larkin AF, Larkin WF, Larkin II WF, et al. Annual torso specific conditioning program for golfers. In: Cochran AJ (ed.) Science and golf: proceedings of the First World Scientific Congress of Golf. London: E. and F.N. Spon, 1990, pp.61-63.

14. Fradkin AJ, Sherman CA and Finch CF. Improving golf performance with a warm up conditioning programme. British Journal of Sports Medicine 2004; 38(6): 762-765.

15. Reyes MG. Maximal static contraction strengthening exercises and driving distance. In: Thain E (ed.) Science and Golf IV: proceedings of the 2002 World Scientific Congress of Golf. London: E and FN Spon, 2002, pp.45-53.

16. Lamberth J, Hale B, Knight A, et al. Effectiveness of a six-week strength and functional training program on golf performance. Int J Golf Sci 2013; 2: 33-42.

17. Fradkin AJ, Sherman CA and Finch CF. How well does club head speed correlate with golf handicaps? J Sci Med Sport 2004; 7: 465-472.

18. Di Francisco-Donoghue J, Werner W and Douris PC. Comparison of once-weekly and twice-weekly strength training in older adults. Br J Sports Med 2007; 41: 19-22.

19. Taaffe DR, Duret C, Wheeler S, et al. Once-weekly resistance exercise improves muscle strength and neuromuscular performance in older adults. J Am Geriatr Soc 1999; 47: 1208-1214.

20. Keogh JWL, Kilding A, Pidgeon P, et al. Effects of different weekly frequencies of dance on older adults' functional performance and physical activity patterns. Eur $J$ Sports Exerc Sci 2012; 1: 14-23.

21. Argus CK, Gill ND, Keogh JW, et al. Effects of a shortterm pre-season training programme on the body composition and anaerobic performance of professional rugby union players. J Sports Sci 2010; 28: 679-686.

22. Cook G, Burton L and Hoogenboom B. Pre-participation screenings: the use of fundamental movements as an assessment of function - part 1. N Am J Sports Phys Ther 2006; 1: 62-72.

23. Frost DM, Beach TAC, Callaghan JP, et al. Using the functional movement screen to evaluate the effectiveness of training. J Strength Cond Res 2012; 26: 1620-1630.

24. Gribble PA, Brigle J, Pietrosimone BG, et al. Intrarater reliability of the functional movement screen. $J$ Strength Cond Res 2013; 27: 978-981.

25. Minick KI, Kiesel KB, Burton L, et al. Interrater reliability of the functional movement screen. J Strength Cond Res 2010; 24: 479-486.

26. Parsonage JR, Williams RS, Rainer P, et al. Assessment of conditioning-specific movement tasks and physical 
fitness measures in talent identified under 16-year-old rugby union players. J Strength Cond Res 2014; 28 : 1497-1506.

27. Kritz M and Cronin J. Static posture assessment screen of athletes: benefits and considerations. Strength Cond J 2008; 30: 18-27.

28. Dalgleish M, Corso B, McTigue $\mathrm{B}$, et al. The golf athlete - ten test-on range golf screening protocol. Brisbane, QLD: The Golf Athlete, 2011, pp.1-24.

29. Horan S, Evans K and Dalgleish M. Reliability of a standardised golf specific musculoskeletal screening protocol designed for use by golf professionals. In: Golf Science. A World Scientific Congress of Golf, Gold
Coast, QLD, 5-7 September 2014, pp.51. Gold Coast, QLD: Griffith University.

30. McGill SM, Childs A and Liebenson C. Endurance times for low back stabilization exercises: clinical targets for testing and training from a normal database. Arch Phys Med Rehabil 1999; 80: 941-944.

31. Robertson SJ, Burnett AF, Newton RU, et al. Development of the nine-ball skills test to discriminate elite and high-level amateur golfers. J Sports Sci 2012; 30: 431-437.

32. Robertson SJ, Burnett AF and Newton RU. Development and validation of the Approach-Iron Skill Test for use in golf. Eur J Sport Sci 2013; 13: 615-621. 